\title{
Using Email Communication to Increase Expatriate Parents' Knowledge of the Human Papillomavirus
}

\author{
Melissa M. Baker ${ }^{1}$, Ratana Somrongthong ${ }^{2}$ \\ ${ }^{1}$ Graduate Institute of International and Development Studies, Genève, Switzerland \\ ${ }^{2}$ College of Public Health Sciences, Chulalongkorn University, Thailand
}

\begin{tabular}{l}
\hline \hline Article Info \\
\hline Article history: \\
Received Nov 20, 2015 \\
Revised Dec 21, 2015 \\
Accepted Jan 10, 2016 \\
\hline
\end{tabular}

Keyword:

Beliefs

HPV

Human Papillomavirus

Knowledge

Vaccination

\begin{abstract}
Expatriates face a unique set of determinants to health which may influence their level of knowledge, perception of available preventative health care alternatives and their health seeking behaviors. The objective of this study is to understand the effect of an email communication intervention on expatriate parents' level of knowledge of the Human Papillomavirus (HPV). Repeated measurement of knowledge was conducted pre- and postintervention among parents who received the study intervention (group 1) and those who received standard care (group 2). Intervention effect was measured by any change in knowledge within and between groups. The group 1 had a significant rise in knowledge mean from baseline to first and then second follow-up ( $m=0.57$ (SD 0.39), $m=0.84$ (SD 0.16) and $m=0.87$ (SD 0.11), respectively). In addition, after receiving the intervention, group 1 felt they had sufficient information to make an informed decision of whether to vaccinate their child(ren), with a significant difference from baseline to first post test, $\left(\chi^{2}(1)=8.50, p<0.05\right)$. Based on an increase in knowledge, the study's email intervention proved effective mode to disseminating HPVrelated information.
\end{abstract}

Copyright (C) 2016 Institute of Advanced Engineering and Science. All rights reserved.

\section{Corresponding Author:}

Melissa M. Baker,

Graduate Institute of International and Development Studies,

Maison de la Paix, Chemin Eugène-Rigot 2, 1202 Genève, Switzerland.

Email: Melissa.baker@graduateinstitute.ch

\section{INTRODUCTION}

The human papillomavirus (HPV) is the most common sexually transmitted infection (STI) in the world [1]. HPV is the leading cause of many persistent infections and afflictions of the sexual and reproductive organs. HPV types 16 and 18 are known to cause $70 \%$ of cases of cervical cancer, and HPV types 6 and 11 are responsible for $90 \%$ of cases of genital warts [2]. Vulnerable populations often have reduced compliance to recommended preventative measures against HPV, including uptake of the HPV vaccine(s) [3], [4]. Due to exposure to unique health risks, expatriates (expats) are a more vulnerable to developing vaccine-preventable infections, such as HPV [5].

Three prophylactic vaccines are available that can prevent HPV. All HPV vaccines are available for females (2vHPV, 4vHPV and 9vHPV) and two vaccines for males (4vHPV and 9vHPV) [6]. The US Centers for Disease Control and Prevention (CDC) recommends that the HPV vaccines be included as a part of routine vaccination for girls and boys age 11 to 12 years, and through to age 26 for females and through age 21 for males who have not be previously vaccinated [7]. Men who have sex with men (MSM) and immunecompromised men through age 26 are also advised to get the HPV vaccine [3].

Despite the availability of preventative measures to protect against this increasingly prevalent sexually transmitted infection, there is continued vaccination resistance against the HPV vaccine [2],[8],[9]. The most persistent and common factors found to influence vaccination resistance include a general lack of 
knowledge of HPV and the HPV vaccine, concerns of vaccine safety and efficacy, and a low perceived susceptibility of contracting HPV [2],[10]-[13].

At the time of this study there was little research that measured expatriate (expat) parents knowledge of HPV and the HPV vaccine, or tested health-information dissemination tools specifically designed to address the gaps in health-related knowledge of transient populations such as expats. Research on parents' vaccination-related knowledge of HPV is important, as some countries require parental consent to vaccinate young people who are below the legal age [9],[14],[15]. Without parental consent it is difficult to vaccinate young people who are below the legal age [14],[16]. Additionally, research on expats is important, as there are an increasing number of individuals and families moving abroad for professional reasons, and for a duration of longer than 6 months at a time [17].

Expats face a unique set of factors that have the potential to create an increased cumulative risk of exposure to vaccine-preventable illnesses in their host country [5],[18]. Many factors increase the likelihood of health risks in moving from developed to less developed countries, including problems adapting to different aspects of their host country (language, climate, politics), culture (status of women, attitudes, religion), and facilities (housing, transportation, infrastructure such as hospitals, police service) [19]. In Bangkok, expats are exposed to a unique set of 1) environmental factors such as congested urban areas and infrastructure, which restrict mobility and access to medical or healthcare, 2) socio-cultural factors such as language barriers and the inability to communicate in Thai to clearly explain health needs or issues, and a lack of neighborhood or community connection which reduces parents' healthy support system, 3) growing political insecurity and violence with recent changes in government which restricts movement in and around the city, 4) greater exposure to high-risk behaviors such as sex tourism, consumption of alcohol, drugs, and cigarettes.

Little information exists about best modes of disseminating health-related information to expats, and about whether mode of communication influences compliance to recommended preventative measures. Expat parents can be difficult to access through traditional channels, therefore this study intervention included email as the mode of disseminating HPV-related information. Of the few studies that have evaluated the effect of email as a health promotion tool, results have been promising, and email has proven to be fast, economical, and convenient [20]-[23].

The purpose of this study was to investigate the preliminary efficacy of an email communication intervention for expatriate (expat) parents living in Bangkok, Thailand, on measures of HPV knowledge. The data generated from this study will determine whether a larger confirmatory study is warranted.

\section{RESEARCH METHOD}

This study was approved by the Ethics Review Committee for Research Involving Human Research Subjects, Health Sciences Group at Chulalongkorn University in December 2012. In November-December 2013, the study was advertised widely in various professional, resource and social networks for expats in Bangkok. Sixty-six parents were recruited. To participate in the study, expat parents had to be from families that relocated abroad for professional reasons, had lived in Bangkok for a minimum of 1 year, be a biological or a non-biological parent or guardian of a child(ren) below the age of 18 years, have not vaccinated their child(ren) with the HPV vaccine(s), proficiency in English (reading and writing), and access to email address, regular use of a computer and Internet. In total, forty-three parents were eligible to participate in the study. Written consent was obtained from all study participants. In total, forty-three parents were eligible to participate in the study.

Parents were randomly allocated to either group $1(n=23)$ or group $2(n=20)$. Group 1 was emailed a series of 12 posters over a period of one month (receiving a new poster three times per week). Each poster contained unbiased, accurate information about HPV or the HPV vaccine that was derived from the World Health Organization, the Public Health Agency of Canada, and the CDC websites. Characteristics from other study's Internet-based health interventions and input from experts were sought and considered throughout this study's intervention design process. 24 Parents from group 2 received the study intervention at the end of the study.

The research team monitored uptake of the intervention by activating an automatic reply tool in the teams' email server, prompting parents to acknowledge receipt of the email prior to viewing the poster, which was sent to the research team email. Upon opening the email the poster was presented as an image within the email.

The study utilized quantitative data measurement tools including a baseline survey, and two followup surveys. The first follow-up survey was administered immediately after the intervention finished. The second follow-up survey was conducted three months after the intervention finished. 
Group 1 and group 2 completed all surveys at the same time and intervals. The survey included questions regarding parents' socio-demographic characteristics and knowledge of HPV and the HPV vaccine. Parents responded 'yes', 'no' or 'do not know' to questions about transmission, prevention, treatment, health effects and outcomes of HPV. Correct responses were scored with 1-point and incorrect responses, 'I do not know' and missing values were scored with no (0) points. Parents were also asked whether they felt they had sufficient information about the HPV vaccine to feel confident in making an informed-decision to give their child the HPV vaccine. The proportion of parents who agreed or disagreed with this statement was measured within groups over time relative to the baseline survey (McNemar Test) and between groups 1 and 2 at each survey period.

Analysis of data was completed using the Statistical Package for the Social Sciences version 21.0 (SPSS, Chicago, Illinois). Statistical tests were performed with 95\% confidence interval (CI) and a p-value $<0.05$ was considered statistically significant. All variables were analyzed for frequencies. Pearson $r$ test was conducted to analyze correlations. Chi-square and t-tests were conducted to test whether there were any differences in the knowledge mean of the group 1 and group 2 at each survey period. A chi-square test was computed to analyze categorical knowledge variables and an independent t-test was computed to explore the knowledge scale.

It should be noted that conclusions from these analyses are made with caution, as the sample size was small as this study was strictly a pilot of an email intervention.

\section{RESULTS AND ANALYSIS}

Socio-demographic data collected at baseline showed no significant differences between study participants. The majority of the parents involved in the study were female $(n=37 ; 86 \%)$ and between the ages of 30 to 45 years $(n=30 ; 69 \%)$. The parents were from fifteen countries of origin, defined as the country from which the parents held a passport, including countries in Asia, North America, Europe, Oceania and Africa. Almost all parents involved in the study were married or living with a partner $(\mathrm{n}=42 ; 97 \%)$. Twentyseven parents $(63 \%)$ reported a religious affiliation and sixteen $(37 \%)$ were unaffiliated. The socio-economic status of the study population was high, with forty parents $(93 \%)$ having completed post-secondary education, and the median annual household income within the range of $\$ 125,000-\$ 149,999$ USD/year. Nineteen parents $(58.7 \%)$ reported a household income $>149,999$ USD/year, and ten parents (23\%) reported an annual household income of $\$ 200,000$ USD or greater/year. There were no statistically significant differences between the socio-demographic characteristics of group 1 and group 2.

Of all parents, $74 \%(n=32)$ had heard of HPV prior to the study. $70 \%(n=32)$ had heard of at least one HPV vaccine, and $37 \%$ of the parents $(n=16)$ had received information that was supporting/recommending the HPV vaccine. Only $42 \%(n=18)$ of parents knew whether their child was in an age group that was eligible for HPV vaccination.

The baseline knowledge scale mean for all parents was low with less than half of the parents answering the questions correctly $(\mathrm{m}=49 \%$; SD0.06). Only $63 \%$ of the parents $(\mathrm{n}=27)$ knew that HPV is a sexually transmitted infection, 65\% ( $\mathrm{n}=28)$ knew that HPV can cause cervical cancer and $42 \%(\mathrm{n}=18)$ knew that HPV could cause genital warts.

Knowledge of HPV prevention and treatment was low with only $19 \%$ of the parents $(n=8)$ reporting that they knew that HPV can go away without treatment. 58\% $(n=25)$ of the parents knew that getting a regular Pap smear examination can reduce a woman's chances of contracting an HPV infection, and only $47 \%(n=20)$ knew that HPV could cause abnormal Pap smear results.

At baseline, there were two categorical knowledge variables that had statistically significant differences between the group 1 and group 2: whether the parent knew if their child was eligible for vaccination at the time of the study $\left(\chi^{2}(1)=6.51, \mathrm{p}=0.05\right)$, and if the parent had heard positive information about the HPV vaccine $\left(\chi^{2}(1)=10.03, \mathrm{p}=0.01\right)$.

Group $1(\mathrm{n}=23)$ mean knowledge scale was low with only $\mathrm{m}=57 \%$ (SD0.39) and the group 2 mean was $\mathrm{m}=39 \%$ (SD0.35). A chi-square test revealed no statistically significant knowledge variable answers of either group 1 or group 2 at baseline. An independent t-test found no differences between the knowledge scale mean of group 1 and group 2.

Correct answers for knowledge variables for all study participants and separated and compared between group 1 and group 2 at first follow-up are captured in Table 2. At first follow-up the knowledge scale mean for group 1 was high $(\mathrm{m}=84 \%$, SD0.17). Group 1 had a high level of knowledge of the health effects of HPV. Of the parents in group 1,95.7\% $(n=22)$ knew that HPV is a sexually transmitted infection that can cause cervical cancer and genital warts. From group 1, 83\% knew that HPV could cause abnormal Pap smear results $(n=19)$. At first follow-up group 1 only $39 \%(n=9)$ knew that HPV can go away without treatment. 
At first follow-up group 2 had a moderate level of knowledge ( $\mathrm{m}=73 \%$, SD0.23). Group 2 had a high level of knowledge of the health effects of HPV, with $100 \%$ of the parents who knew that HPV can cause cervical cancer $(n=20), 80 \%$ knew that HPV is an STI that can cause genital warts $(n=16)$. 90\% of group 2 knew that getting regular Pap test could reduce a woman's chances of getting cervical cancer $(n=18)$. From group 2, 60\% $(\mathrm{n}=12)$ of the parents knew that HPV can cause abnormal Pap smear results and $25 \%$ $(n=5)$ of the parents knew that HPV can go away without treatment.

A Chi-Square test revealed no statistically significant knowledge variable responses of group 1 and group 2 at first follow-up. Knowledge questions were then merged into a knowledge scale. The Levene's test for equality of variances was computed to determine differences between group 1 and group 2 knowledge scale means - no statistically significant differences were found; therefore a pooled t-test was computed. A statistically significant difference was found between the group 1 and group 2 knowledge scale mean $(\mathrm{t}(41)=2.91, \mathrm{p}<0.01)$. This statistic implies that there is evidence that being exposed to the study intervention could have affected level of knowledge. Cohen's $d$ and $r 2$ were computed to measures the effect size. The Cohen's d effect size was 88.76 and r2 was 0.17 , which means a large effect (almost $89 \%$ ) with $17 \%$ of the variance of knowledge level accounted for from receiving the study intervention (being in group 1) at first follow-up.

At second follow-up the knowledge scale mean for group 1 was high $(\mathrm{m}=83 \%$, SD0.12). Group 1 knowledge of the health effects of HPV was high. All parents in group $1(100 \%)$ knew that HPV is a sexually transmitted infection that can cause genital warts, and 96\% (SD0.21) knew that HPV can cause cervical cancer. 96\% (SD0.21) of group 1 knew that HPV can cause abnormal Pap smear results, and by getting regular Pap smears this can reduce a woman's chances of getting cervical cancer. Group 1 had a low level of knowledge that HPV can go away without treatment (35\%, SD0.49).

At second follow-up the group 2 knowledge scale mean was moderate ( $\mathrm{m}=64 \%$, SD0.22). Group 2 had a high level of knowledge of the health effects of HPV, with 75\% (SD0.44) who knew that HPV is an STI. From group 2, 90\% knew that HPV could cause cervical cancer and genital warts (SD0.31). 90\% of group 2 knew that getting regular Pap test can reduce a woman's chances of getting cervical cancer (SD0.31), and 70\% knew that HPV can cause abnormal Pap smears (SD0.47). In group 2, knowledge that HPV can go away without treatment was low (10\%, SD0.31).

A chi-square test revealed no statistically significant knowledge variable responses of group 1 and group 2 at second follow-up. Knowledge variables were calculated into a knowledge scale and computed with an independent t-test to determine whether there were any differences between group 1 and group 2 knowledge scale means at second follow-up. The Levene's test for equality of variances indicated that the variances were not to be assumed as equal $(\mathrm{F}=4.4 ; \mathrm{p}=0.04)$; therefore we used the statistical outputs from the Welch $t$ test results. A statistically significant difference was found $(\mathrm{t}(41)=3.44, \mathrm{p}<0.01)$. The measurements for effect size were high with Cohen's $\mathrm{d}=107.43$ and $\mathrm{r} 2=22.4$. These data indicate that $22.4 \%$ of the variance in the knowledge level could be accounted from receiving the study intervention (being in the group 1) at second follow-up.

In each survey, parents were asked whether they had sufficient information about HPV and the HPV vaccine to make a decision of whether to administer the vaccine to their child(ren). The proportion of parents who agreed or disagreed with this statement was measured within groups over time relative to the baseline survey (McNemar Test) and between groups at each survey period.

For group 1, no significant change was observed between baseline and first follow-up ( $\mathrm{p}=0.18$ ). There was also no significant difference between baseline and second follow-up $(p=0.23)$. Nevertheless, there were a higher proportion of parents who agreed that they had sufficient information in the follow-up surveys $(70 \%)$ relative to the baseline survey $(48 \%)$.

As for group 2, no significant differences were observed between the baseline and first follow-up $(\mathrm{p}=0.50)$. There was however a slight increase in the proportion of parents who felt they had sufficient information at first follow-up (15\% at baseline and $25 \%$ at first follow-up). Significant differences were found between baseline and the second follow-up responses $(p<0.10)$, with a significant difference in the total proportion of parents who felt they had sufficient information at second follow-up survey (45\%) relative to the baseline (15\%).

The comparison between the group 1 and group 2 at each survey period highlighted some differences between the two groups. Two-thirds of parents agreed with the statement in the baseline survey, although a significantly larger proportion were from the group $2\left(\chi^{2}(1)=5.25, \mathrm{p}<0.05\right)$. The effect size for this difference is moderate $(\varphi=0.35)$, however. At first follow-up, the difference was much more pronounced with nearly half of the parents disagreeing with the statement, with over two-thirds of the parents from the group 1 group compared to only a quarter from the group 2 . This result is statistically significant $\left(\chi^{2}\right.$ $(1)=8.50, p<0.05)$ and has a larger effect size relative to the baseline $(\varphi=0.45)$. Finally, by second follow-up, the difference was no longer statistically significant $\left(\chi^{2}(1)=2.65, \mathrm{p}=0.103\right)$. 


\section{CONCLUSION}

This pilot study corroborates findings from many other studies. First, consistent with findings from other HPV vaccination resistance studies, we found that expat parents had a low level of knowledge of HPV and the HPV vaccine at baseline [24]-[27]. Overall knowledge of HPV [at baseline] was low $(m=49 \%$, SD0.38). Second, similar to other studies that have evaluated the effect of sexual and reproductive health educational interventions and found correlations between increased knowledge and positive behavioral change [28],[29], we found that expat parents feel more confident making informed-decisions about their child's health, including deciding whether to vaccinate with the HPV vaccine, when they have sufficient information.

This brings us to our last remark that a larger confirmatory study is recommended, and should measure the effect of the email-based educational intervention on knowledge and behavioural change (vaccination uptake). This study provides evidence that email may be a successful mode of disseminating health-related information. These data are consistent with data found in other intervention studies that measured the effect of email as a mode of disseminating health-related information [20],[22],[30].

There are many limitations to this study. The study aimed to include a diverse study sample of expat parents living in Bangkok, Thailand, however, as the socio-demographic data reveals, the study sample is not representative of all expats living abroad, as this study sample is comprised of expats with high socioeconomic status (SES). The study participants were also highly-educated, the majority of whom having a minimum college or university degree. This reduces the generalizability of the findings to a wider population of expats who live and work away from their country of origin. Despite this potential limitation, the study sample was a diverse group of expat parents. Study participants were self-selected which may also reduce the generalizability, as the sample may be representative of expat parents who are interested in learning about sexual health. Future studies can be expanded to include a wider sample of expat parents who live in different parts of the world.

With growing evidence of the HPV vaccines efficacy and recommended use in boys and girls, it is critical that parents be informed of the susceptibility, severity and consequences of HPV, and the benefits and barriers of getting the HPV vaccine, so that they can make informed decisions of whether to vaccinate their child prior to sexual debut. Researchers have worked to address the factors that influence parental vaccination intention and uptake by implementing interventions specifically aimed at educating parents about sexual health, HPV and the HPV vaccine [31]-[33]. This study contributes to existing literature and intervention research on the barriers to parents' uptake of the HPV vaccine. In considering other studies and preventative interventions, online access to vaccine information and services is ideal for populations who face unique social and environmental determinants of health, including expats who relocate their families and careers abroad. Evidence suggests that people who have email communication with a health professional or online (Internet) access to health information are more educated about their medical decisions, compared to those who do not have online access [34]. In an effort to design a program that facilitates convenient delivery of reliable HPV education through the use of Internet-based technology, we created an email communication intervention that aims to equip expat parents with the knowledge to make informed decisions of whether to vaccinate their child.

The low levels of knowledge reported in this study emphasize the need for HPV educational programs for expat parents in order to improve HPV vaccination intention and uptake. The data compiled here within should be used with caution, and help to inform the development of an HPV educational program aimed at increasing expat parents' knowledge and improving perception, and measure whether these variables influence HPV vaccination intention and vaccination uptake. This intervention and others should aim to address some of the salient barriers to vaccination intention and uptakes, which were revealed in this study will include knowledge of HPV transmission, prevention and health effects, and HPV vaccine efficacy, safety and potential side effects.

As hoped, this study provides useful information that warrants further research using email as a mode to disseminate to communicate HPV-related information to expats. With an increasing number of persons moving abroad for professional reasons, it is critical that expat parents have access to health information that provides them confidence and the ability to make informed-decisions and influence preventative, healthy behaviors.

\section{ACKNOWLEDGEMENTS}

This work was supported by the Higher Education Research Promotion and National Research University Project of Thailand, Office of the Higher Education Commission (grant number AS1148A-55).

All procedures performed in studies involving human participants were in accordance with the ethical standards of the institutional and/or national research committee and with the 1964 Helsinki 
declaration and its later amendments or comparable ethical standards. This article does not contain any studies with animals performed by any of the authors.

\section{REFERENCES}

[1] World Health Organization, "Sexually Transmitted Infections (STIs)", 2013. Available at http://www.who.int/mediacentre/factsheets/fs110/en/ Accessed 3 July 2015.

[2] Holman D., Benard V., Roland K., Watson M., Liddon N., Stokley S., "Barriers to human papillomavirus vaccination among US adolescents: a systematic review of the literature", JAMA Pediatr, vol/issue: 168(1), pp. 7682, 2014.

[3] Hariri S., "HPV Vaccination Update - 2015", National Center for HIV/AIDS, Viral Hepatitis, STD and TB Prevention, CDC, 2015. Available at http://www.cdc.gov/vaccines/ed/ciinc/downloads/2015-0520/NCIRDwebinarMay2015.pdf Accessed 3 July 2015.

[4] Pierre CM., Lim PL., Hamer DH., "Expatriates: Special Considerations in Pretravel Preparation", Curr Infect Dis Rep, vol. 15, pp. 299-306, 2013.

[5] Dijkestra JA., Chappuis F., Loutan L., "Vaccine-preventable diseases in long-term expatriates", J Travel Med, vol. 12, pp. S47-S57, 2005.

[6] Slomski A., "New HPV Vaccine provides wider protection", JAMA, vol/issue: 313(16), pp. 1609, 2015.

[7] Centers for Disease Control and Prevention, "HPV vaccination", 2014. Available at http://www.cdc.gov/vaccines/vpd-vac/hpv/ Accessed 12 December 2014.

[8] Allen JD., De Jesus M., Mars D., Tom L., Cloutier L., Shelton RC., "Decision-making about the HPV vaccine among ethnically diverse parents: Implications for health communications", J Oncol, 401979, 2012.

[9] Batista Ferrer H., Trotter C., Hickman M., Audrey S., "Barriers and facilitators to HPV vaccination of young women in high-income countries: a qualitative systematic review and evidence synthesis", BMC Pub Health, vol. 14, pp. 700, 2014.

[10] Javanbakht M., Stahlman S., Walker S., et al., "Provider perceptions of barriers and facilitators of HPV vaccination in a high-risk community", Vaccine, vol/issue: 30(30), pp. 4511-4516, 2012.

[11] Reynolds D., O'Connell KA., "Testing a model for parental acceptance of human papillomavirus vaccine in 9- to 18-year-old girls: a theory-guided study", J Pediatr Nurs, vol/issue: 27(6), pp. 614-625, 2012.

[12] Dorell C., Yankey D., Kennedy A., Stokley S., "Factors that influence parental vaccination decisions for adolescents, 13 to 17 years old: National Immunization Survey-Teen, 2010”, Clin Pediatr (Phila), vol/issue: 52(2), pp. 162-170, 2013.

[13] Drolet M., Bénard É., Boily MC., Ali H., et al., "Population-level impact and herd effects following human papillomavirus vaccination programmes: a systematic review and meta-analysis", The Lancet Infectious Diseases vol/issue: 15(5), pp. 565-580, 2015.

[14] Wood F., Morris L., Davies M., Elwyn G., "What constitutes consent when parents and daughters have different views about having the HPV vaccine: qualitative interviews with stakeholders", $J$ Med Ethics, vol/issue: 37(8), pp. 466-471, 2011.

[15] Cooper Robbins SC., Bernard D., McCaffery K., et al., “'I just signed”: Factors influencing decision-making for school-based HPV vaccination of adolescent girls", Health Psychol, vol/issue: 29(6), pp. 618-625, 2010.

[16] Brown K., Kroll J., Hudson M., Ramsay M., Green J., et al., "Factors underlying parental decisions about combination childhood vaccinations including MMR: A systematic review", Vaccine, vol/issue: 28(26), pp. 42354248, 2010.

[17] Patel D., Easmon C., Seed P., et al., "Morbidity in expatriates - a prospective cohort study", Occup Med, vol. 56, pp. 345-52, 2014.

[18] Shepherd SM., Shoff WH., "Vaccination for the expatriate and long-term traveler", Informa Healthcare, Expert Review of Vaccines, vol/issue: 13(6), pp. 775-800, 2014.

[19] Jones S., "Medical aspects of expatriate health: health threats", Occup. Med, vol/issue: 50(8), pp. 572-578, 2000.

[20] Lim MSC., Hocking JS., Aitken CK., et al., "Impact of text and email messaging on the sexual health of young people: a randomized controlled trial”, Journal of Epidemiology \& Community Health, vol. 66, pp. 69-74, 2012.

[21] Kothe EJ., Mullan BA., "Acceptability of a theory of planned behavior email-based nutrition intervention", Health Promotion International, vol/issue: 29(1), pp. 81-90, 2014.

[22] Constantino RE., "Comparing face-to-face and email intervention for intimate partner violence (IPV) survivors: Technologies influencing nursing”, 26 ${ }^{\text {th }}$ International Nursing Research Congress, 23-27 July 2015, San Juan, Puerto Rico.

[23] Brouwer W., Kroeze W., Crutzen R., et al., "Which intervention characteristics are related to more exposure to internet-delivered healthy lifestyle promotion interventions? A systematic review", Journal of Medical Internet Research, vol/issue: 13(1), pp. e2, 2011.

[24] Reiter PL., Brewer NT., Gottlieb S., McRee AL., Smith JS., "Parents' health beliefs and HPV vaccination of their adolescent daughters", Social Science \& Medicine, vol/issue: 69(3), pp. 475-480, 2009.

[25] Sadaf A., Richards JL., Glanz J., Salmon DA., et al., "A systematic review of interventions for reducing parental vaccine refusal and vaccine hesitancy", Vaccine, vol/issue: 31(40), pp. 4293-4304, 2013.

[26] Fishman J., Taylor L., Kooker P., "Parent and adolescent knowledge of HPV and subsequent vaccination", Pediatrics, vol/issue: 134(4), pp. 2013-3454, 2014

[27] Fu LY., Bonhomme LA., Chenoa CS., et al., "Educational interventions to increase HPV vaccination acceptance: A systematic review", Vaccine, vol/issue: 32(17), pp. 1901-1920, 2014. 
[28] Poorman E., Gazmararian J., Parker RM., et al., "Use of text messaging for maternal and infant health: A systematic review of the literature", Maternal and Child Health Journal, vol/issue: 19(5), pp. 969-989, 2015.

[29] Pazol K., Zapata LB., Tregear SJ., et al., "Impact of contraceptive education on contraceptive knowledge and decision-making: A systematic review”, American Journal of Preventative Medicine, vol/issue: 49(2), pp. S46-S56, 2015.

[30] Wantland DJ., Portillo CJ., Holzemer WL., et al., "The effectiveness of Web-based vs. Non-Web-based interventions: A meta-analysis of behavioral change outcomes", J Med Internet Res, vol/issue: 6(4), pp. e40, 2004.

[31] Kennedy A., Sapsis KF., Stokley S., et al., "Parental Attitudes Toward Human Papillomavirus Vaccination: Evaluation of an Educational Intervention, 2008", Journal of Health Communication, vol. 16, pp. 300-313, 2011.

[32] Krawczyk A., Lau E., Perez A., et al., "How to Inform: Comparing Written and Video Education Interventions to Increase Human Papillomavirus Knowledge and Vaccination Intentions in Young Adults", Journal of American College Health, vol/issue: 60(4), pp. 316-322, 2012.

[33] Spleen AM., Kluhsman BC., Clark AD., et al., "An Increase in HPV-Related Knowledge and Vaccination Intent Among Parental and Non-Parental Caregivers of Adolescent Girls, Age 9-17 Years, in Appalachian Pennsylvania", J Canc Educ, vol. 27, pp. 312-319, 2012.

[34] Odone A., Ferrari A., Spagnoli F., et al., "Effectiveness of interventions that apply new media to improve vaccine uptake and vaccine coverage", Hum Vacc Immunother, vol/issue: 11(1), pp. 72-82, 2015. 